OPLS-DA modelling with an optimised threshold diagnosed IgG4-RD - as opposed to PSC - with an accuracy of $86 \%$, sensitivity of $96 \%$, and specificity of $70 \%$ (figure 1), adjusted for age, gender, comorbidities, serum IgG4 level and medications. Both IgG4-RD and PSC were independently distinguishable from $\mathrm{HC}$ with an accuracy of $96 \%$ and $91 \%$, respectively. When only IgG4-SC patients $(n=23)$ were included with large-duct PSC patients $(n=81)$, the accuracy was $88 \%$. When IBD was excluded as a comorbid condition (IgG4-SC $\mathrm{n}=20$, PSC $\mathrm{n}=22$ ), the diagnostic AUC was 0.998 (0.991-1.000).

The metabolomic signature determined by serum NMR in patients with IgG4-RD and more specifically IgG4-SC, is distinct from PSC and $\mathrm{HC}$ in our cohort. Metabolomic profiling has the potential to be incorporated as an additional criterion to improve the diagnosis of IgG4-RD and help distinguish IgG4-SC from PSC.

\section{OPPORTUNITIES TO INTERVENE AFTER EARLY DETECTION OF ALCOHOL RELATED LIVER DISEASE}

Tiwonge Jason Nyirenda*, Lucy Turner, Rebecca Bishop, lan Rowe, Richard Parker. Leeds Teaching Hospitals NHS Trust, Leeds, UK

\subsection{6/gutjnl-2021-BASL.5}

Alcohol related liver disease (ALD) is common worldwide and a frequent cause of ill health and death, particularly amongst younger people. We have previously shown that histological progression is slow. We examined the clinical progression of ALD and how interventions following detection of liver disease could alter the natural history.

The electronic health records of patients with ALD were examined retrospectively to identify: time of first liver blood tests (LBT), first abnormal LBT, time of diagnosis of liver disease and first episode of decompensation of liver disease. Decompensated liver disease was defined as: ascites, variceal bleeding, hepatic encephalopathy, alcoholic hepatitis or hepatorenal syndrome. Attendance at alcohol services and/or hepatology services was noted. Baseline characteristics including laboratory and anthropometric data were recorded. Patient's postcode was used as a measure of deprivation. Data were analysed with Kaplan Meier survival analysis and Cox proportional hazard analysis, using R. All patients gave informed consent for their records to be accessed as part of the ALLHEAL study.

Seventy-three patients with decompensated liver disease were analysed. The median delay from the detection of disease (i.e. first abnormal LBT) to decompensated ALD was 6.6 years (95\% CI 4.7 - 10.1 years). In Cox proportional hazard analysis, independent factors that influenced time from detection to decompensation were: age, bilirubin, albumin, INR, platelets and mean cell volume. Engagement with alcohol services was the strongest predictor of time to decompensation: hazard ratio $0.50(0.31-0.80, \mathrm{p}=0.004)$. The median time to decompensation in patients who engaged with alcohol services was 10.3 years $(8.6-12)$ versus 3.9 years $(1.3-6.6)$ in those who did not $(\log$ rank $\mathrm{p}<0.001)$. Excluding patients with decompensation within 3 months of first abnormal LBT did not change the pattern of results but statistical significance was lost.

The natural history of ALD allows for a significant period of time between detection and liver-related morbidity.
Attendance at alcohol services is associated with a significant delay between detection to decompensation in this cohort of patients. These data can support efforts to detect liver disease amongst persons with alcohol use disorder.

\section{AMINOBISPHOSPHONATES ENHANCE LIVER-RESIDENT GAMMA DELTA T CELLS FOR EFFICIENT TARGETING OF HEPATOCELLULAR CARCINOMA}

${ }^{1}$ Nekisa Zakeri ${ }^{*},{ }^{2}$ Andrew Hall, ${ }^{1}$ Leo Swadling, 'Laura J Pallett, ${ }^{1}$ Nathalie M Schmidt, ${ }^{1}$ Mariana 0 Diniz, ${ }^{1}$ Stephanie Kucykowicz, ${ }^{1}$ Oliver E Amin, ${ }^{3}$ Amir Gander, ${ }^{4}$ Massimo Pinzani, ${ }^{3}$ Brian R Davidson, ${ }^{2}$ Alberto Quaglia, ${ }^{1}$ Mala K Maini. ${ }^{1}$ Division of Infection and Immunity, University College London, UKi ${ }^{2}$ Department of Cellular Pathology, Royal Free London NHS Foundation Trust, UK; ${ }^{3}$ Division of Surgery, University College London, UK; ${ }^{4}$ Institute for Liver and Digestive Health, Royal Free London NHS Foundation Trust, UK

\subsection{6/gutjpl-2021-BASL.6}

Background More effective immunotherapeutic strategies are urgently needed for hepatocellular carcinoma (HCC). Gamma delta $(\gamma \delta)$ T-cells are attractive candidates for cancer immunotherapy due to their potent cytotoxicity, tissue localisation and HLA-unrestricted tumour reactivity. We characterised liver and tumour infiltrating $\gamma \delta$ T-cells in HCC, and explored whether modulating features of tissue-residency could provide a novel immunotherapeutic approach.

Methods Lymphocytes isolated from paired blood, liver, and tumoural tissue from patients with HCC $(n=31)$ in comparison to colorectal cancer liver metastases $(n=30)$ were analysed by multiparameter flow cytometry. $\gamma \delta$ T-cell counts were determined by immunostaining. Long-lived persistence of intrahepatic $\gamma \delta$ T-cells was examined using donor and recipient HLA-mismatched liver allografts (7-11 years post liver transplantation). Aminobisphosphonate (Zoledronic acid, ZOL) and IL-2 expanded blood V82 Tcells, intrahepatic lymphocytes, and tumour-infiltrating lymphocytes, were co-cultured with human hepatoma cell-lines (HepG2, HuH7) pre-treated with ZOL to promote

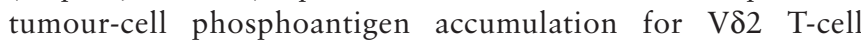
receptor activation.

Results Higher intratumoural $\gamma \delta$ T-cell counts were associated with smaller HCC tumour size and greater 3-year patient survival $(\mathrm{p}<0.01) . \gamma \delta$ T-cells exhibited a tissue-resident memory (TRM) phenotype $(\mathrm{CD} 69+\mathrm{CD} 49 \mathrm{a}+)$ in human liver and HCC, with superior anti-tumour cytokine production and long-lived persistence in the liver ( $>10$ years), an attractive profile to recapitulate with immunotherapy. A subset of $\gamma \delta \mathrm{T}$ cells, V82 T-cells, were selectively depleted within HCC but displayed the highest $\gamma \delta$ TRM phenotype. In vitro expansion of blood V $\delta 2$ T-cells using clinically approved ZOL and IL-2 induced a de novo TRM phenotype with improved cytotoxicity. Furthermore, direct sensitisation of hepatoma cell-lines with ZOL enhanced the anti-tumour function (IFN $\gamma, \mathrm{TNF} \alpha$ ) of co-cultured expanded V $\delta 2$ T-cells and V $\delta 2$ TRM cells isolated from HCC livers and tumours, with a significant increase in tumour-cell lysis.

Conclusion Liver-resident $\gamma \delta$ T-cells possess beneficial and long-lived immunotherapeutic properties. Our findings indicate a novel immunotherapeutic strategy for HCC, combining the use of aminobisphosphonates to induce $\gamma \delta$ TRM for potential adoptive cell transfer, with intra-tumoural delivery to sensitise HCC for more efficient $\gamma \delta$ T-cell based targeting. 